\title{
Effects of oxygen radicals in low-pressure surface-wave plasma on sterilization
}

\author{
Masaaki Nagatsu, ${ }^{a)}$ Fumie Terashita, and Hiroyuki Nonaka \\ Graduate School of Science and Engineering, Shizuoka University, 3-5-1 Johoku, \\ Hamamatsu 432-8561, Japan \\ Lei Xu \\ Graduate School of Electronic Science and Technology, Shizuoka University, 3-5-1 Johoku, \\ Hamamatsu 432-8561, Japan \\ Toshi Nagata and Yukio Koide \\ Hamamatsu University School of Medicine, 1-20-1 Handayama, Hamamatsu 431-3192, Japan
}

(Received 12 October 2004; accepted 4 April 2005; published online 18 May 2005)

\begin{abstract}
The effects of oxygen radicals on sterilization were studied using a $2.45 \mathrm{GHz}$ surface-wave oxygen plasma. A population of $1.5 \times 10^{6}$ Bacillus stearothermophilus spores was irradiated for 3 min or more with oxygen plasma, generated at pressures between 6 and $14 \mathrm{~Pa}$. The decimal reduction value ( $D$ value), a measure of the effectiveness of sterilization, was determined to be about 15-25 s. Using only oxygen radicals, excluding all charged particles, the $1.5 \times 10^{6}$ spores were sterilized with a $D$ value of $30-45 \mathrm{~s}$ after 5 min or more of irradiation. On scanning electron microscopy, the length and width of the spores changed significantly due to chemical etching by oxygen radicals. (C) 2005 American Institute of Physics. [DOI: 10.1063/1.1931050]
\end{abstract}

The methods used in the sterilization of medical instruments depend on the heat resistance of the instruments. Traditionally, for heat resistant instruments, either dry heat or hot steam sterilization is used, while, for heat sensitive instruments, sterilization with ethylene oxide is used. The disadvantage of the dry heat or hot stream sterilization is that they can only be applied to instruments that can withstand the high temperatures. The disadvantages of ethylene oxide gas sterilization are that ethylene oxide is toxic and poses environmental risks. As well, it takes one or more weeks for the ethylene oxide levels to return to safe levels, which is much longer than the time required to sterilize the equipment. Another type of sterilization method, which combines hydrogen peroxide gas with an rf discharge plasma, is commercially available. ${ }^{1}$ Currently, this equipment is assumed to effectively sterilize due to the high concentration of hydrogen peroxide rather than to the plasma component.

At present, various low-temperature plasma sterilization techniques have been developed, such as low-pressure glow discharges, ${ }^{2}$ glow discharges at atmospheric pressure, ${ }^{3-5}$ downstream plasma produced by microwave excitation, ${ }^{6-8}$ moving atmospheric microwave plasmas, ${ }^{9}$ and surface-wave plasmas. ${ }^{10,11}$ The plasma sterilization method has certain advantages over conventional methods, including a low sterilization temperature (often less than $70^{\circ} \mathrm{C}$ ) and a short sterilization period of several minutes to $1 \mathrm{~h}$.

Previously, we reported that a six-log reduction in spores could be achieved after only a 3 min irradiation with a lowpressure oxygen surface-wave plasma. ${ }^{11}$ In this study, we studied the effect of oxygen radicals on sterilization.

The experimental setup used has been described by Nagatsu et al. ${ }^{11}$ The $2.45 \mathrm{GHz}$ microwave guided by a rectangular waveguide was fed into the stainless-steel chamber (diameter of $40 \mathrm{~cm}$ and height of $40 \mathrm{~cm}$ ) filled with oxygen gas

${ }^{a)}$ Electronic mail: tmnagat@ipc.shizuoka.ac.jp through slot antennae cut in the broad face of each waveguide. ${ }^{12}$ The launched microwave power varied from 0 to $1.5 \mathrm{~kW}$. The vacuum chamber's pressure was lowered using a turbomolecular pump to a pressure of an order of $10^{-3} \mathrm{~Pa}$. We compared the sterilizing characteristics of oxygen, hydrogen, and argon discharges at low pressures of about 6-14 $\mathrm{Pa}$ and a gas flow rate of $200 \mathrm{sccm}$. A population of $1.5 \times 10^{6}$ Bacillus stearothermophilus spores (ATCC No.12980) was placed on small stainless-steel disks and used as the biological indicator. ${ }^{13}$ The disks were placed in Petri dishes set onto a substrate stage with a diameter of $200 \mathrm{~mm}$ about $15 \mathrm{~cm}$ below the quartz vacuum window. A microwave having an incident power of $750 \mathrm{~W}$ was launched into the chamber in order to produce the plasma. The plasma irradiation period varied from 0 to $10 \mathrm{~min}$. The stage temperature was measured using a thermocouple attached below the metal stage and thermolabel sheets attached on the rear surface of the Petri dish. After plasma irradiation, the spores were incubated in culture tubes for 7 days at an incubation temperature of $55-60{ }^{\circ} \mathrm{C}$ as is standard for Bacillus stearothermophilus.

Figure 1 shows the colony count results of the Bacillus stearothermophilus spores irradiated with oxygen plasma. As shown in Fig. 1, the plotted data roughly lie on a straight line. From the slope of the line, we calculated the decimal reduction values ( $D$ value) to be approximately $15-25 \mathrm{~s}$. When the plasma irradiation lasted for more than $3 \mathrm{~min}$, it was found that the number of colony forming units was zero. In Fig. 1, we also plotted the inverse of the results of the experiments that yielded a zero spore count at $2.5 \mathrm{~min}(0.5)$ and at $3 \mathrm{~min}(0.1)$. Furthermore, to compare the effect of gas species on sterilization, we tested argon and hydrogen plasmas using the same discharge condition: A microwave power of $750 \mathrm{~W}$, a pressure of about $10 \mathrm{~Pa}$, and irradiation duration of $10 \mathrm{~min}$. However, as shown in Fig. 1, for both the argon and hydrogen plasma discharges, the spores survived even after a 10 min irradiation. From this, we can deduce that 


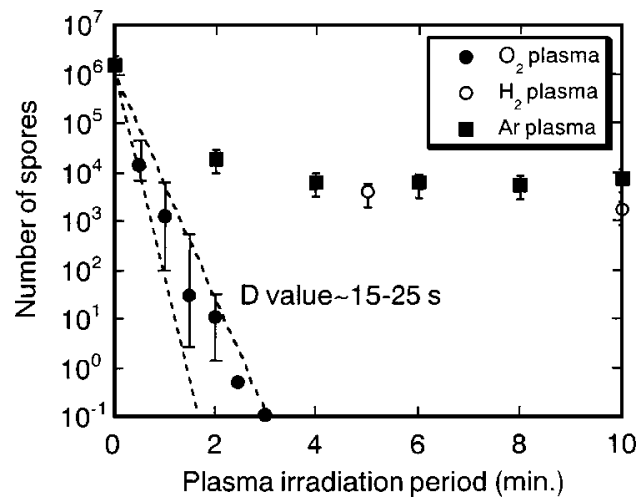

FIG. 1. Survival curves of the B. stearothermophilus spores irradiated by the oxygen, hydrogen, and argon surface-wave plasmas.

electrons and ions are not the primary agents in this sterilization process. Consequently, it might be the oxygen plasma that plays a role in killing the spores in this experiment. It should be noted that the stage temperature, based on the thermocouple attached below the metal stage and the thermolabel sheets attached on the rear surface of the Petri dish, was always less than $70{ }^{\circ} \mathrm{C}$ for all of the different gas species. Figure 2 shows the change in the stage temperature as measured using the thermocouple versus the plasma irradiation periods for the different gas species of $\mathrm{Ar}, \mathrm{H}_{2}$, and $\mathrm{O}_{2}$. After a 10 min plasma discharge, the temperature increased by approximately $25{ }^{\circ} \mathrm{C}$ in all cases. Based on the thermolabel measurements, it was also shown that the surface temperature of the Petri dish was less than $55^{\circ} \mathrm{C}$ after a $10 \mathrm{~min}$ plasma discharge.

To study the shape and size of the spores before and after plasma irradiation, we carried out measurements using a scanning electron microscope (SEM). It was shown that the length and width of the spores dramatically decreased after only a 4 min plasma irradiation. The ellipsoidal spores with a length of $1.5 \mu \mathrm{m}$ and a width of $0.9 \mu \mathrm{m}$ shrank to jellybeanlike slender shapes. ${ }^{11}$ This could be attributed to chemical etching by the oxygen radicals generated in the oxygen plasma. However, in the case of the argon or hydrogen plasma, it was found that the sizes and shapes of the spores were not affected by a $10 \mathrm{~min}$ plasma irradiation.

In order to examine the effect of the UV radiation emitted from the oxygen plasma, we placed a thin quartz plate with a thickness of $3 \mathrm{~mm}$ over the Petri dish to block any

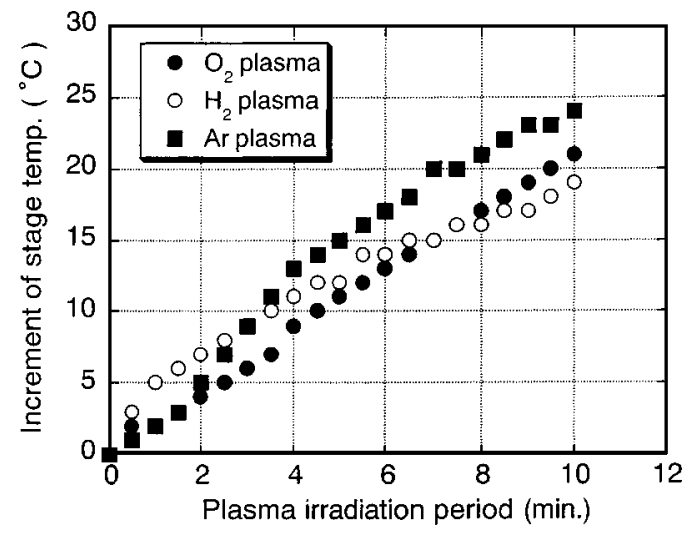

FIG. 2. The change in the stage temperature measured with the thermocouple vs the plasma irradiation periods for the different gas species: oxygen, hydrogen, and argon. a 10 min oxygen radical irradiation.
Downloaded 10 Jul 2008 to 133.70 .80 .50 . Redistribution subject to AlP license or copyright; see http

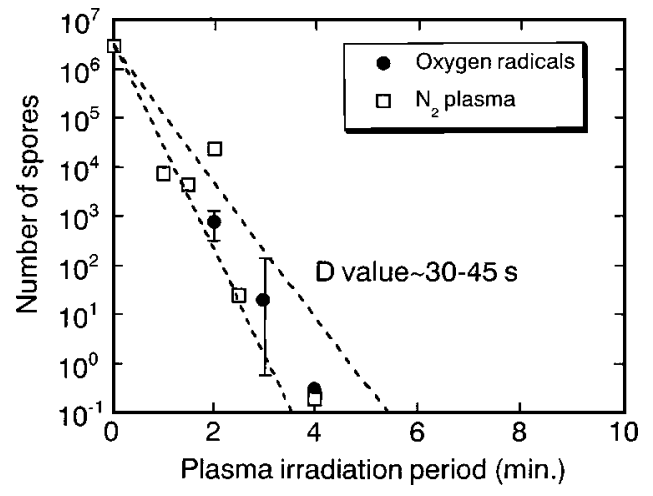

FIG. 3. Survival curves of the B. stearothermophilus spores irradiated with oxygen radicals and nitrogen surface-wave plasmas..

charged or neutral particles, while only UV photons with a wavelength, $\lambda$, greater than $160 \mathrm{~nm}$ were able to penetrate the quartz plate. However, after a 10 min oxygen plasma discharge, the spores were still alive and had not changed their size or shape. From these results, it would appear that UV radiation is not necessary for this sterilization to be effective in the present experiment. However, it could be the case that, as the radicals approached the Petri dish, UV photons might be generated. To further investigate the role of UV photons, future experiments using optical emission spectroscopy need to be performed.

To examine the effect of oxygen radicals on sterilization, we placed a stainless-steel plate with $4 \mathrm{~mm}$ diameter holes on a stage located $8 \mathrm{~cm}$ below the quartz window. Although the electrons and ions were blocked by this punched plate, the neutral radicals could pass through the plate, together with any UV photons that might have a weak effect on sterilization. We measured the survival curve of the Bacillus stearothermophilus spores in a Petri dish located $7.5 \mathrm{~cm}$ below the punched plate. In Fig. 3, the number of colony form-

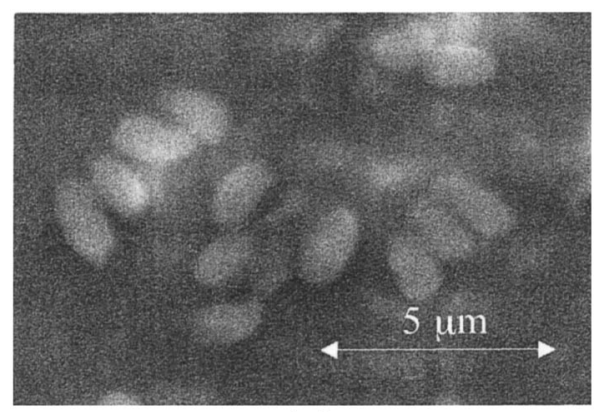

(a)

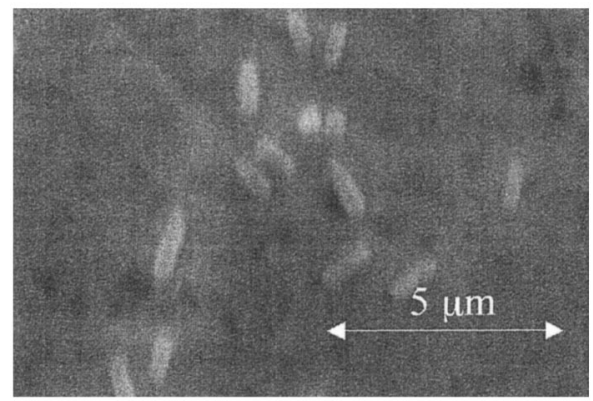

(b)

FIG. 4. SEM images of B. stearothermophilus spores (a) before and (b) after

a 10 min oxygen radical irradiation. 
ing units for different plasma discharge periods is shown. From the slope of the survival curve, we found the $D$ value to be roughly 30-45 s. The SEM images of the original spores are shown in Fig. 4(a), while the images after a 10 min radical irradiation are shown in Fig. 4(b). As in previous experiments, it can be seen that the spores changed their shape and size. ${ }^{11}$ Hence, we concluded that the primary sterilization species were the oxygen radicals produced by the surface-wave sustained oxygen plasma.

Recently, we performed an analogous experiment using nitrogen plasma discharge in order to study its effect on sterilization. From a colony count, we found that the spores were sterilized after a 4 min discharge. A preliminary result of the colony count is also plotted in Fig. 3, where the $D$ value was roughly estimated as $30-40 \mathrm{~s}$, although there is insufficient data for conclusive results. Furthermore, it is interesting to note that the spore shapes were almost identical to the original ones, which differs from the findings with oxygen plasma or oxygen radical irradiation. This result could possibly be caused by UV photons emitted by the nitrogen plasma. Similar experiments have been done by Moisan's group in the microwave plasma with $\mathrm{N}_{2}-\mathrm{O}_{2}$ gas mixtures and they showed that UV emission ranging from 250 to $320 \mathrm{~nm}$ was strongly enhanced by adding $2 \%$ of $\mathrm{O}_{2}$ gas content into $\mathrm{N}_{2}$ plasma. ${ }^{7,8}$ To confirm the role of UV photons emitted directly from the plasma or those generated when the radicals approach the Petri dish, an examination using UV emission spectroscopy is planned, and we hope to report the results in the near future.

In conclusion, we studied the effect of oxygen radicals produced in a surface-wave plasma discharge on sterilization. Experimental results showed that the Bacillus stearothermophilus spores were sterilized at a low temperature by oxygen plasma irradiation after $3 \mathrm{~min}$ or more or by oxygen radical irradiation after $5 \mathrm{~min}$ or more. We also tested argon and hydrogen plasma discharges using the same discharge conditions and found that sterilization was not achieved even after a 10 min plasma discharge. From these empirical re- sults, it might be concluded that the sterilization mechanism in the oxygen plasma is due to a chemical etching reaction from the reactive oxygen radicals. Further studies will be required to examine the role of UV emission in nitrogen plasma discharges.

This work has been supported by Grants-in-Aid for Scientific Research of JSPS, partly by the regional new consortium projects of METI, and by a Grant of the RSP program of the Shizuoka Organization for Creation of Industries.

${ }^{1}$ P. T. Jacobs and S. M. Lin, in Disinfection, Sterilization, and Preservation, 5th ed., edited by S. S. Block (Lippincott, Williams, and Wilkins, Philadelphia, PA, 2000), Chap. 38, pp. 747-763.

${ }^{2}$ I. A. Soloshenko, V. V. Tsiolko, V. A. Khomich, A. I. Shchedrin, A. V. Ryabtsev, V. Y. Bazhenov, and I. L. Mikhno, Plasma Phys. Rep. 26, 792 (2000).

${ }^{3}$ T. C. Montie, K. Kelly-Wintenberg, and J. R. Roth, IEEE Trans. Plasma Sci. 28, 41 (2000).

${ }^{4}$ M. Laroussi, I. Alexeff, and W. L. Kang, IEEE Trans. Plasma Sci. 28, 184 (2000).

${ }^{5}$ V. Y. Bazhenov, A. I. Kuzmichev, V. I. Kryzhanovsky, I. L. Mikhno, A. V. Ryabtsev, I. A. Soloshenko, V. A. Khomich, V. V. Tsiolko, and A. I. Shchedrin, in Proceedings of the International Symposium on Plasma Chemistry, Orleans, France, 2001, Vol. II, pp. 3005-3010.

${ }^{6}$ M. Moisan, J. Barbeau, S. Moreau, J. Pelletier, M. Tabrizian, and L'H. Yahia, Int. J. Pharm. 226, 1 (2001).

${ }^{7}$ N. Philip, B. Saoudi, M. C. Crevier, M. Moisan, J. Barbeau, and J. Pelletier, IEEE Trans. Plasma Sci. 30, 1429 (2002).

${ }^{8}$ M. Moisan, B. Saoudi, M. C. Crevier, N. Philip, E. Fafard, J. Barbeau, and J. Pelletier, Proceedings of 5th International Workshop on Microwave Discharges: Fundamentals and Applications, Greifswald, Germany, 8-12 July, 2003, pp. 210-221.

${ }^{9}$ J. Ehlbeck, A. Ohl, M. Maas, U. Krohmann, and T. Neumann, Surf. Coat. Technol. 174, 493 (2003).

${ }^{10}$ S. Lerouge, M. R. Wertheimer, R. Marchand, M. Tabrizian, and L'H. Yahia, J. Biomed. Mater. Res. 51, 128 (2000).

${ }^{11}$ M. Nagatsu, F. Terashita, and Y. Koide, Jpn. J. Appl. Phys., Part 2 42, L856 (2003).

${ }^{12}$ M. Nagatsu, T. Sano, N. Takada, T. Hirao, and H. Sugai, Jpn. J. Appl. Phys., Part 2 39, L929 (2000).

${ }^{13}$ Raven Biological Laboratories, Omaha, NE, Instruction Manual of Raven Biological Laboratories, Inc. ATCC No. 12980. 